\title{
Developing a mobile activity game for stroke survivors—lessons learned
}

\author{
Charlotte Magnusson ${ }^{1}$ (1) $\cdot$ Kirsten Rassmus-Gröhn ${ }^{1} \cdot$ Bitte Rydeman $^{1}$
}

Received: 14 February 2020 / Accepted: 14 July 2020 / Published online: 23 July 2020

(c) The Author(s) 2020

\begin{abstract}
Persons who have survived a stroke might lower the risk of having recurrent strokes by adopting a healthier lifestyle with more exercise. One way to promote exercising is by fitness or exergame apps for mobile phones. Health and fitness apps are used by a significant portion of the consumers, but these apps are not targeted to stroke survivors, who may experience cognitive limitations (like fatigue and neglect), have problems with mobility due to hemiplegia, and balance problems. We outline the design process, implementation and user involvement in the design of an exergame app that is specifically targeted to stroke survivors, and present the lessons learned during the design process.
\end{abstract}

Keywords Stroke $\cdot$ Activity $\cdot$ Game $\cdot$ Interaction $\cdot$ Design

\section{Introduction}

For persons who have survived a stroke, making conscious changes in their life-style becomes important; both to aid recovery and to avoid additional strokes. There are health and fitness related risk factors connected to stroke incidence (c.f. [1]) that have to do with hypertension, blood pressure, diabetes, smoking and cardiovascular disease. Health and fitness apps for the general public is a growing market of great importance. According to [2], there were about 259.000 health and fitness apps available on the major app stores in 2016. Health and fitness apps are used by $33 \%$ of the consumers and wearable technology by $21 \%$ of the consumers [3] (2016). Users of health apps and wristbands explicitly state that they use the apps to keep better track of their condition or keep them healthy, and the most used app types relate to fitness and nutrition/diet. Even so, life-style change is hard, and this kind of technology suffers from high rates of abandonment [4].

Charlotte Magnusson

charlotte@certec.lth.se

Kirsten Rassmus-Gröhn

kirre@certec.lth.se

Bitte Rydeman

bitte.rydeman@certec.lth.se

1 Department of Design Sciences, Lund University, P.O. Box 118, 22100 Lund, Sweden
There are many location based games on the market (a recent overview can be found at [5]), but these are designed for the general population and are not tailored for stroke survivors. As stroke survivors are often older (average age 69 in 2005 [6]), and cognitive problems are a common sideeffect of a stroke, many mainstream apps can be too hard to use. For example, screens are often filled with content and interaction can be quite complex or stressing. For apps using step goals, pre-set goals for daily steps may not be appropriate. Any app for this group has to work even if you are only able to walk shorter distances, since many stroke survivors have partial hemiplegia and balance problems.

Given the scarcity of studies on mobile games for stroke survivors, our work has been exploratory in order to learn more about how an activity game designed for stroke survivors should be designed. A first report on our design was published in [7], an outline of the development can be found in [8]. The present paper briefly describes the design process, presents the final game designs and presents and discusses evaluation results.

\section{Related work}

Studies on the design of mobile apps using different visualizations to promote physical activity have been carried out for some time. In an early study, Fish 'n'Steps [9] showed physical activity as changes in fish in a fish tank. Another set of 
studies [10] used first a shareable fitness journal (Houston) followed by a glanceable display where your activity would translate into a growing flower garden (UbiFit). Although the term game is used by the authors of [9], these works primarily deal with the visualization of progress and activities. The main lessons learned from this work were: supporting the cognitive activation of health goals, encouraging a range of behaviours, focusing on longer term patterns and facilitating social support as an "optional but not primary motivator".

Behaviour change is a field of research of its own. Designers of persuasive technology typically draw on theories for behaviour change; [11] proposes design strategies based on a combination of behavioural and social psychological theories. In addition, self-determination theory (SDT) can provide an understanding of motivation in relation to exercise/physical activity. In a systematic review [12], it is suggested that valuing the outcome of the exercise is important for the initial adoption, while the actual experience of the exercise is more important for long term maintenance. It is pointed out that it can even be counterproductive to focus too much on the utility/outcome of exercising, since this may inadvertently reduce the experiential rewards of being physically active.

Games, in contrast with activity monitoring, focus on experiential qualities. Suitably designed video games have been used in rehabilitation, e.g. [13] and there are studies on how mobile devices can support rehabilitation exercises [14]. With Pokémon Go, it became apparent that location based games can be powerful tools for encouraging activity, also among persons who normally were less interested in physical activities [15]. The success of Pokémon Go has led to the development of a plethora of different mobility games, but mobility games specially designed for our particular user group, stroke survivors, still appear to be missing. The mobility app closest to a game we have been able to find is the activity tracker STARFISH [16], an app designed for stroke survivors, which makes use of a social setting and where the progress of a group of participants is visualized as properties of fish in a fish tank.

Both UbiFit, Pokémon Go and STARFISH rely heavily on visual interaction, as do most apps and games, also in the rehabilitation domain. An example is the interactive tiles for balance training during stroke rehabilitation by Donker et al. [17], which showed that providing real time visual feedback improved motivation and was helpful for balance rehabilitation.

Still, auditory feedback via sonification has been seen to be helpful for improving motoric skills [18]. Stienstra et al. [19] studied how movement sonification improves proprioception during speedskating, if it is latency-free, and the richness of data details generated by the movement are preserved in the richness of sound parameters, such as dynamics, loudness, tempo, pitch and timbre. Other relevant work has proven the benefits of movement sonification on proprioception and awareness [20, 21]. Moreover, music and rhythm therapy are proven to help stroke survivors increase the feeling of being connected to their own body. A study by Thornberg et al. [22] shows that music and rhythm exercises were considered positively challenging for stroke survivors, while facilitating motor planning and coordination. Music is also a significant motivation factor while training which can give emotional pleasure [22]. Pleasant sounds tend to persuade the user into behavioural change [19].

While it is known that haptic/tactile feedback can improve both task performance and promote a sense of social presence [23], tactile interaction design for stroke survivors is less explored. Touch in the form of tangible interaction has been shown to be valuable for users with cognitive disabilities-Caltenco and Larsen [24] found tangible interaction supported effective interaction in sensory stimulation environments for persons with severe developmental disabilities (Snoezelen). Vandermaesen et al. [25] developed a tangible table top game to support lifting and left/right movement skills. However, they evaluated only with stroke rehabilitation nurses and not with stroke survivors themselves. The presented work was done in parallel with the project ActivAbles, where tangible interaction for stroke survivors was explored for balance and hand/upper arm training (tangible designs with visual and auditory feedback) [26].

\section{Design process}

The mobile game developed is part of a larger collection of exergames and self-help tools connected to two research projects, STARR (EU) and ActivAbles (NordForsk). In these projects, requirements and ideas were captured by interviews, focus group discussions and workshops with stroke survivors, informal carers and medical personnel [8, 27, 28]. Requirements that were seen as particularly relevant to the mobile activity game were:

- Ease of use (but not childish)

- One-handed use (due to hemiplegia)

- Possible to personalize (e.g. left- or righthandedness)

- Make it multimodal (vision-hearing-touch, for different abilities)

- Cater for fatigue (not overwhelm the user)

- Safety (since it should be possible to use outdoors)

- Native language use (second languages are often lost after stroke)

- Adapt game content frequency and placement to the abilities of the user 
Technically oriented requirements were that the game should be possible to play anywhere, also indoors, and that the user should be able to track both activity and game progress (in case these were different).

With these baseline requirements, some inspiration was taken from games like Pokémon Go and Ingress as well as an inclusive, location based, game for visually impaired children [29]. For the game to be possible to use anywhere, we decided to rely on step-counts, and not location. Positioning/ location accuracy is still a problem indoors, except in spaces specially equipped for indoor positioning. Furthermore, in location based applications, there is always a risk of ending up in an area without content. As the game is a mobile game, the initial target user group was stroke survivors who were able to move independently and able to use a smartphone. To promote movement, and also make the game easier to use with one hand, games were designed to primarily make use of the movement of the whole device.

The development was explorative and iterative, involving 18 persons ( 2 researchers and 16 stroke organization members) aside from the developer, as illustrated by the Fig. 1. A variety of game designs were introduced in the mini-games in order to explore the design space and gather user feedback. The designs were updated iteratively based on the feedback received from the user testing. The user involvement is described more elaborately in the section Testing process.

\section{Game design}

An in-depth description of the game can be found in [7], the main points are repeated in the following. The game is a walking game implemented for the iPhone, in which you can select if you want to track your steps, your distance or your active time. A step counting algorithm based on peak detection in Kalman filtered accelerometer data was implemented, since to be able to base gaming element on walking, it was important that users get real-time feedback on their movement. The algorithm worked surprisingly well compared to the built in step counting, but cannot be

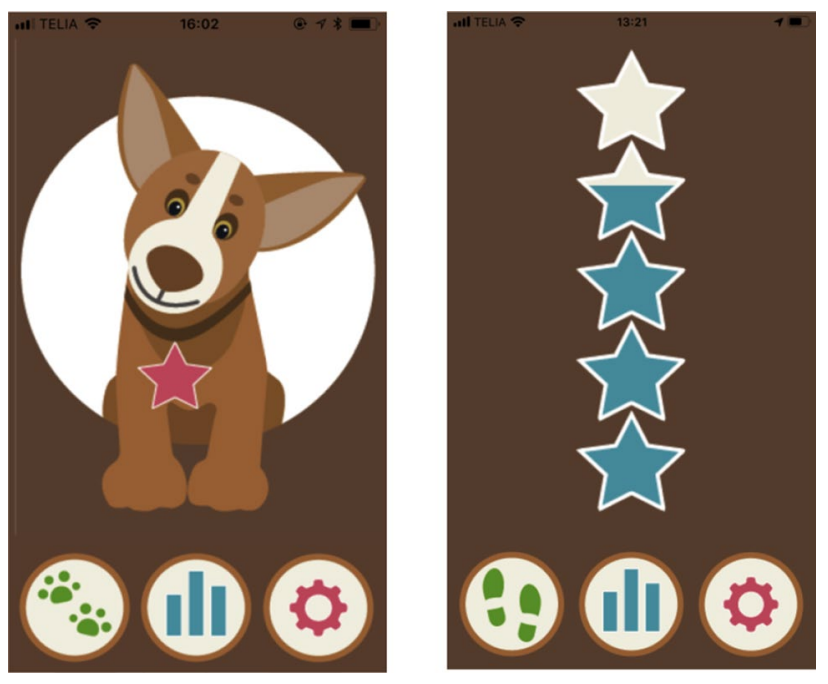

Fig. 2 Left: entry screen (dog theme). Right: entry screen (star theme)

expected to be as accurate over longer periods of time. Thus, to avoid accumulated errors, the step count is compared to step data from Apple Health and corrected so that the total step count agrees with apple health. For distance tracking, a combination of GPS, and when GPS is poor or unavailable, a distance estimate based on the step count times the average distance per step is used. Like for the step count, the total distance is compared to the distance found in Apple Health and corrected. Active time is measured as the time when the app detects the phone is moving. The active time for when the app isn't actively used during the day is approximated through step count times average time per step.

The current version of the game contains three different game designs. The first is a game built on mini-games that appear in a semi random fashion. The second version of the game is a game where keys and chests are automatically picked up while walking, and once you have finished walking, the keys can be used to unlock the chests. The third version of the game takes you on a visual journey. Each completed walk takes you one step further on the journey. Game versions 2 and 3 are "scaled down" based on user
Fig. 1 Schematic representation of iterative process. Red circles are tests, and the figure shows the number of users involved. Yellow circles are development periods. The blue circle represents idea generation and requirements capture which involved several user and codesign activities. The names of the phases are used below

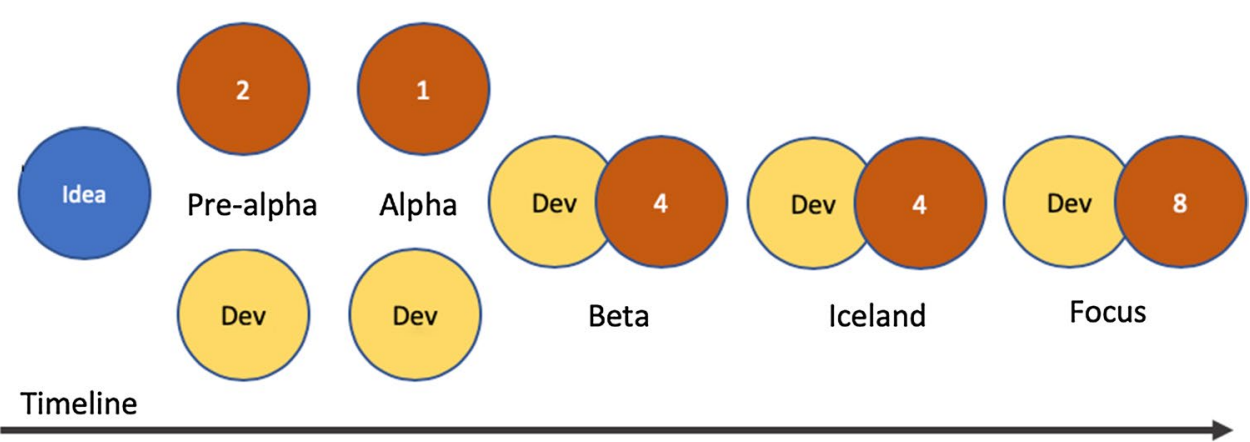



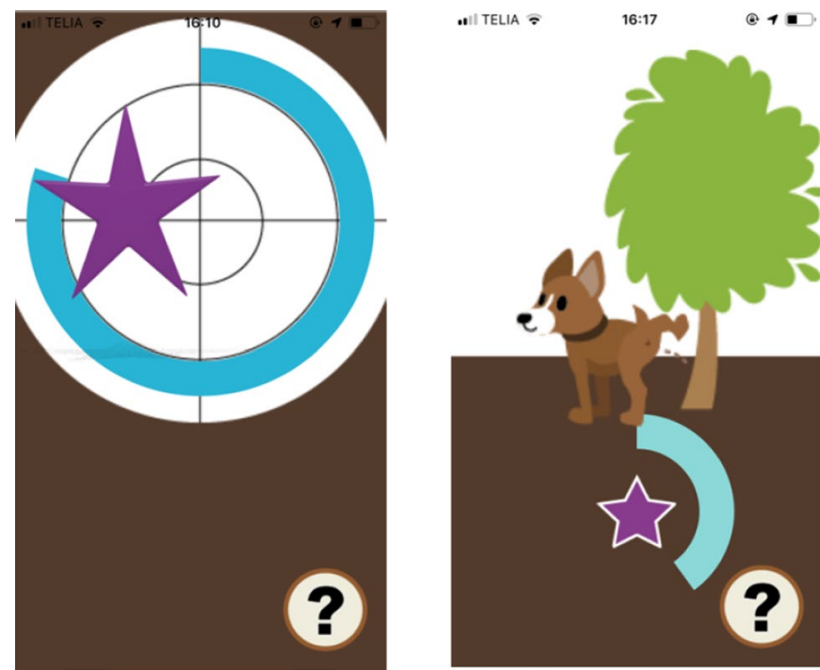

Fig. 3 Screen shot of the pointing mini game, making use of the compass (left: star theme, right: dog theme)
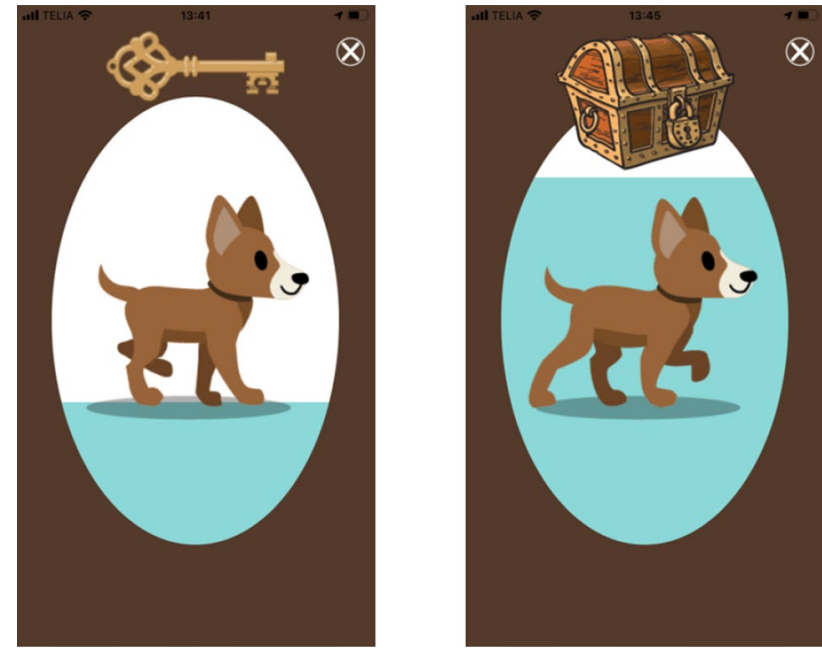

Fig. 4 Picking up keys and chests during a walk

feedback implying interaction while walking can be "too much", and require no user interaction at all during walking.

The walking app has two themes (Fig. 2), and allows the user to select the one they prefer. The underlying mechanics of the games are the same, but the sounds and graphics are different in the two versions (Fig. 3).

The app has settings that allow you to select the game version and theme, and to customize the game difficulty of the mini-games, furthermore it also allows the complete removal of game components so that the app becomes an activity monitoring app.

During a walk the app provides progress feedback by filling up an elongated ellipse on the screen (see Fig. 4 under game version 2). On top of this is either an animated walking dog or animated footsteps which runs as long as the app detects the user is moving. The game can give a notification when the user is half-way to their goal, and also gives a notification once the goal is reached. These notifications are given through on screen information as well as repeated vibrations and sounds. The game will also give sound and vibration feedback and ask for confirmation when the user is about to exit the game.

\subsection{Game version 1: mini-games}

The game notifies the user of the presence of a mini game by a star "falling" over the screen from top to bottom. The user is also notified through repeated sounds and vibrations. The mini game is launched by pressing the star. The star "falls" as long as the user walks, but stops if the user stops, making it easier to catch the star. These mini games make use of different gestures with the whole phone-pointing, tilting, shaking - but also walking faster. The mini games are based on gestures/movements made with the whole phone, minimizing screen interaction, to simplify one-handed and outdoor use while on the move, and are described in detail in [8]. There are currently six different mini games, pointing with the phone, walk faster, jerking/throwing gesture, balancing the phone, tilting and tilt and shoot.

\subsection{Game version 2: key and chests}

In this game version you pick up keys and chests as you walk. What object you pick up on each occasion is randomly assigned. When an object is picked up the phone vibrates, and a sound is heard: for a key a clinking sound, and for a chest a "thud", allowing you to keep the phone in your pocket during the walk and still get feedback of your progress. The number of objects picked up during a single walk is constant (10) and independent of the length of the walk.

Once a walk is completed you can see your results and use the keys to unlock the chests to see how much treasure you have collected (Fig. 5).

\subsection{Game version 3: journey}

The third and final game version is scaled down even further, and (inspired by the importance of anticipation and curiosity in UbiGreen [30]) takes you forwards through a visual journey. The game requires little interaction, you need to start a walk by pressing a button, and when the walk is completed you progress one step in the journey. As with all the game versions you can also get a half-way reminder. This version of the game is currently purely visual and consists 
Fig. 5 Left: result screen. Middle: chest overview. Right: unlock a chest by clicking on the keys

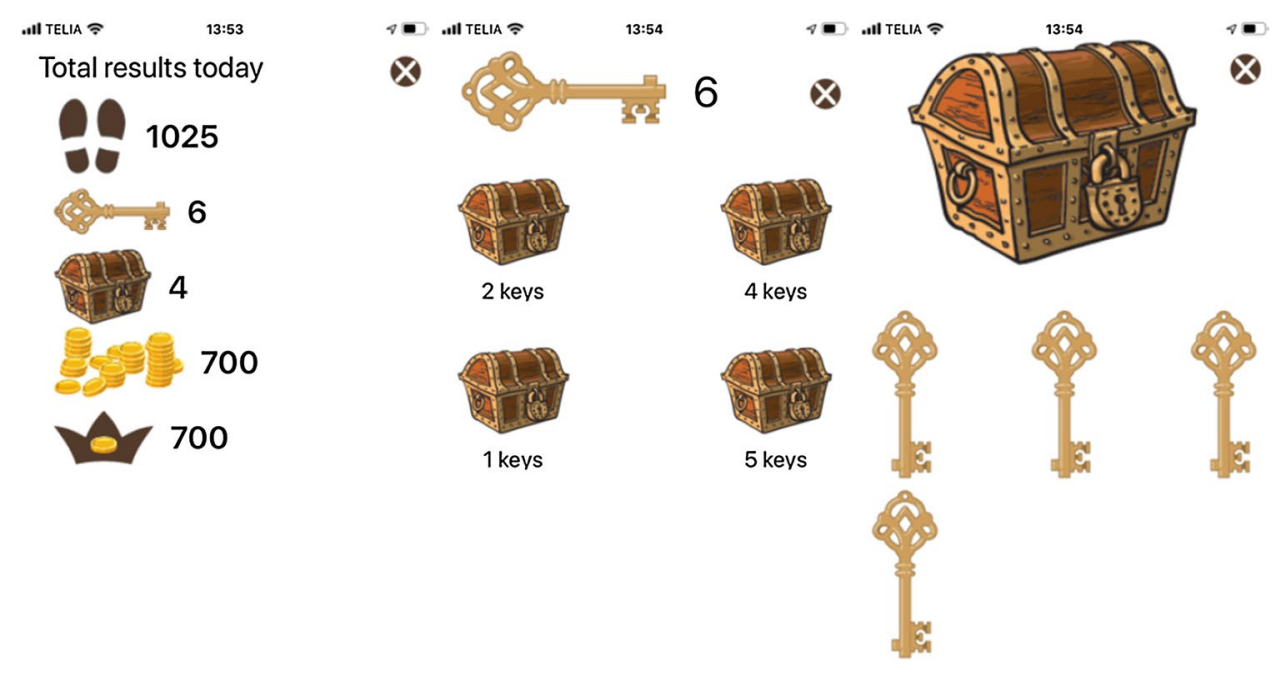

Fig. 6 The balloon moving through three "stops" in a pyramid landscape, and then reaching a forest

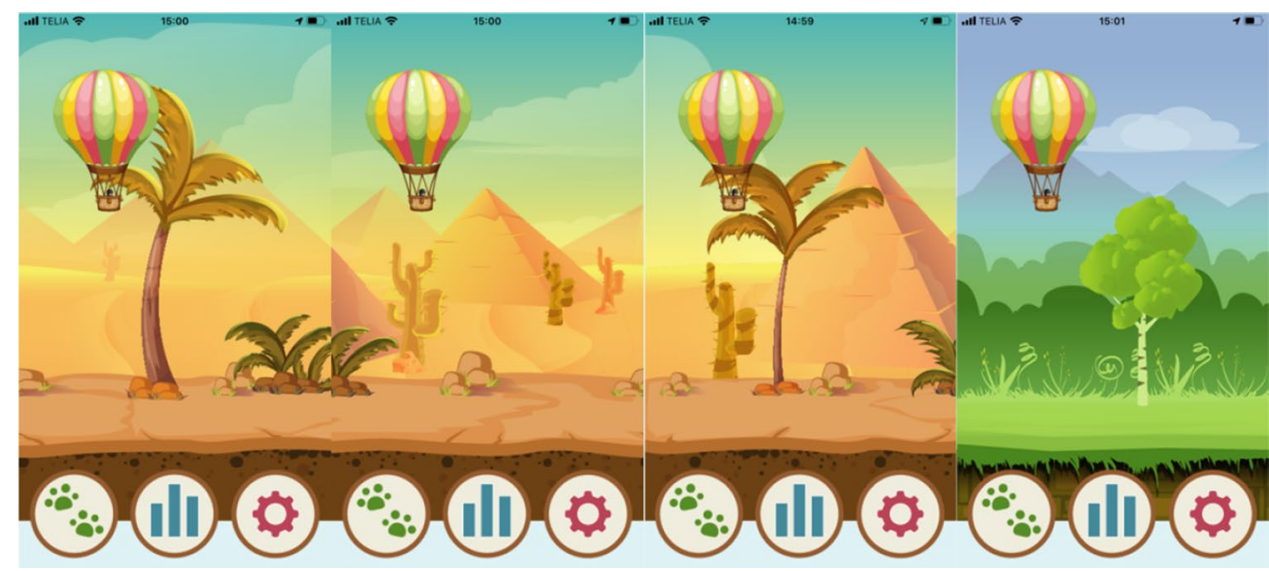

of a balloon moving through different landscapes. There are three "stops" in each landscape, then you move to a new landscape. If successful, the story could be made auditory in future versions of the app (Fig. 6).

\subsection{Audio and vibration design}

The initial design was inspired by popular location based games (e.g. Pokémon Go) for the notion of mini-games, as well as by the interaction in a proof of concept game for visually impaired children [29], where the user interacts with the game by moving the whole phone instead of doing gestures on the screen. Gestures were selected to explore game designs involving both whole body movement (walking faster, rotating the whole body) as well as hand/arm gestures (tilt and shake). Feedback in the games was designed to be multimodal in order to allow the user to rely on different senses.

An important aspect of the game was that it should be possible to keep in the pocket while walking. Thus all feedback given when the user was on the move, had to be given via sounds and/or vibrations. Following [31], distance or closeness to a goal in game version 1 was mapped either to pitch or to volume. In the star theme mini game feedback was generally mapped to musical notes with varying pitches, while in the dog theme some mappings were changed to dog barks of varying volume. In game version 2 symbolic sounds (clink or thud), indicate if you have picked up a key or a chest. Game version 3 currently has no specific sound feedback except general alarms/ reminders.

For alarm type notifications the star theme used clear "ping" sounds, while for the dog version the pings were combined barks to keep the clarity of the pings, while still provide a feeling of the dog notifying you. In the dog version, the app makes small noises (e.g. panting while walking, small woofs to acknowledge button presses in the main menu) to heighten the feeling of interacting with a dog.

Vibration is used mainly as alarm/notification. Important events, such as a star appearing in game version 1, the half way reminder and the end of a game walk, are 
all accompanied by longer, repeated, vibration pulses designed to be hard to miss. Less important events, e.g. the appearance of a key or a chest, are accompanied by single, shorter, vibration pulses.

\section{Testing process}

The first prototype of the step counting game was tested first by the team, to ensure the basic functionality worked (pre-alpha tests). After this we contacted a stroke survivor from the Malmö stroke organization (male, age 62), who was willing to alpha test the app. This tester would send almost daily reports with opinions and suggestions which allowed us to improve and further develop the functionality, and in principle functioned as a co-designer.

Once all the mini games in the step counting game worked reliably four beta testers were recruited: three men and one woman, ages between 43 and 68. All alpha and beta testers had some remaining problems after their stroke, with walking, with balance and with brain fatigue. We visited each of the new testers in their home, installed the app and made initial settings and provided a written "getting started" guide and a paper manual. We also did a demo walk together with the user, to ensure he or she was able to use the app. The results from this testing reported in [7].

During a feasibility study in Iceland, four users tested the app (one woman, three men, ages between 63 and 79). The results of this testing is reported in [32]. Based on the feedback from the feasibility study, the new games versions 2 and 3 were added to the app.

To date, the last activity has been a focus group with 8 participants held at the Malmö stroke organization. Participants were 3 men, 5 women, ages 59-82, one wheelchair and one walker user, of which 6 were stroke survivors and 2 were informal carers/family. The focus group consisted of a groupwise test, with a following focus group discussion with all participants present. One of the participants was the original alpha tester who did not participate in the group test since they had already played all game versions. The other participants were all new to the game. Each group $(2+2+3$ participants) was accompanied by a researcher and tested the different game versions (mini games, keys and chests, journey) in randomized order. The games were played during a walk in the neighborhood that lasted roughly $45 \mathrm{~min}$. The researcher made observations and asked participants to comment on their experience (think aloud) while walking. Since earlier testers had commented negatively on the dog, the original star theme was used during the focus group. The activity ended with a group discussion where all 8 participants were present.

\subsection{Summary of alpha, beta and Icelandic test results}

The results from the alpha and beta testing together with the Malmö stroke organization [7] were mixed. The feedback varied, the alpha tester enjoyed the game and kept testing for several months. This tester enjoyed the challenges, and found level progression with new and challenging/changing mini games an important motivator. The beta testers were less positive. While all were able to use the game at the introduction session, they didn't use it much voluntarily. One of the users "felt stupid" doing the games in public, while two users reported the game element being "too much", while still potentially finding games like this interesting. We also got feedback indicating the original star theme was "boring", something which led to the development of the "dog theme". The testers in Iceland tested the game [32] with the dog theme. They enjoyed seeing their step count progress, but found the games overwhelming. One participant also found the dog theme "childish".

\subsection{Test results, focus group}

The results of the focus group differed from the results obtained from several of the previous tests. Participants were generally positive to the app and thought the games were fun. The test groups consisted of (1) two stroke survivors with almost no walking difficulties, (2) one stroke survivor with a walker, one stroke survivor who mainly had fatigue and problems with motivation, and one informal carer who was not used to walking long distances and (3) a person in a wheelchair and their spouse. All three groups tested all versions. To reduce order effects, the groups tested the game versions in different orders. In the groups, all persons played each version at least once.

The first group were most impressed by the mini-games version, and thought the other versions had too little interaction. They had little to comment on the use and did not experience any problems. In the second group, issues such as the difficulty of hearing the audio feedback was brought forward, in particular when the phone was put in the pocket. The person with the walker had difficulties playing the mini games and handling the walker, in particular the pointing mini game. The tester with fatigue pointed out that all three versions could be interesting, depending on the daily shape. Maybe you had a slight headache and needed to take a walk but the mini-games were too much, while they could be very motivating another day. In the third group, the person in the wheelchair was not able to walk and the wheelchair was pushed by the spouse (or the test leader when the spouse tested the app). In principle, this meant that the person was not actively walking or moving. For example, while playing the pointing mini-game, someone else had to rotate the 
wheelchair. The other mini-games (except the catching gesture) were played successfully. The spouse had a tendency to dizziness, which meant that the pointing mini-game was a challenge. The most important results from this group were, that even if they could not do all the activities in the game, it was still meaningful and gave a sense of accomplishment, and they enjoyed playing the game together. The person in the wheel chair also payed particular attention to the game feedback (audio and visual) in e.g. the keys and chests version.

During the focus group discussion with all groups and the alpha tester present, all game versions were said to be useful. A new issue was also brought forward, that the fact that you had to stop walking to carry out the mini games could interfere with the "flow" of walking. It was possible to use the games both with a walker and a wheelchair, but it was not without problems-dealing with both the phone and an aid while walking can be a challenge. In contrast with the earlier negative opinions on the dog, several participants now thought the dog cute, and would like to try it.

\section{Discussion}

In this section we discuss the lessons we have learned during our design process. Many of these relate to the design, but some also to the design process.

Support varying user abilities Stroke survivors are a diverse group. The results of our testing brings this home, and coupled with the fact that many stroke survivors are vulnerable and used to failing, it becomes a true challenge to develop games that provides a suitable challenge, while at the same time allowing people to succeed. Part of the diversity is also the fact that abilities may vary from day to day-what is a reasonable challenge a "good" day, may be much too hard on a "bad" day. This makes it important to develop a range of different games, allowing people to pick and choose. It also has implication for standard game elements as leveling and increased difficulty - these need to be forgiving, to allow for abilities that vary over time.

Taste varies Aesthetics is important, but taste varies. What one person sees as childish, another may see as cute. This makes recommendations such as "designs should not be childish" [28] hard to follow in practice, and aesthetics that can be personalized might be one solution.

Design for multimodality The audio and sound design has appeared to work as intended generally, allowing users to keep the phone in the pocket. For users who have hearing problems, or don't like to use the sounds, the phone can be held in the hand or kept in a mitten, with vibrations providing notifications. A specific challenge in the mini games has been to reflect the effects of gesture interaction in audio. In the games we have used distance mapped both to pitch and volume [31]. While this could potentially have been done with musical mappings, we had design requirements pointing to a need for simplicity [28]. Thus, we tried to keep our feedback simple and have limited it to distance mappings combined with success and failure information. Although not formally tested, it is our impression is that the pitch design is clearer. While the volume mapping is quite intuitive - things in the real world tend to get louder as they are closer-you don't get much information when the distance is longer and the volume thus very low. An observation specific for the dog theme made by one of our users, is that real dogs in the environment may respond to dog sounds coming from the game when sounds are played from the loudspeaker. We also note, that even though the games are designed so as to not require the user to look at the screen while walking, most people still do this—at least occasionally.

An interesting observation from the app design process, is that while one can come up with a wide range of gesture based challenges, it can be a pedagogical challenge to come up with an explanation of what the user needs to do in order to succeed in the game. As an example: While the jerking gesture in itself is not complicated, providing appropriate feedback supporting the learning of the gesture, together with an appropriate mental model for the user of what you should do, required several iterations in the early game versions.

Avoid sensory overload An important point for many stroke survivors is to avoid sensory overload. The activity of walking (or any other physical/social activity) may be hard enough for the user. Thus care needs to be taken when designing games and feedback, in order to allow users to personalize feedback, but also allow flexibility in the timing - e.g. allowing the user to interact actively during pauses or after the activity. While it appears we have succeeded reasonably in allowing users to select when they interact, we note that even the limited multimodal feedback used in the mini-games, appears to have been too much for some of our test users.

A guiding principle for all our designs has been that the interaction should be flexible to allow users with different perceptual and cognitive abilities to use the developed technology. Thus, we have included designs which make use of multimodal feedback - visual, auditory and haptic (vibration). This comes with two issues: (1) too much simultaneous information may overwhelm the user. It is common for stroke survivors to have brain fatigue, which makes multimodality a potential problem. (2) to avoid overwhelming the user, one could use settings. But settings in themselves run 
the risk of overwhelming the user. So far, we have opted for multimodality as our basic approach, but added a limited number of settings.

It should be noted that the technology restricts what you are able to do. UbiFit [10], used a glanceable display, something which is impossible on the iPhone. A potential solution to this is to add connected objects able to provide information at a glance. The feedback lights described in [32] were intended to fill the same role, and have gotten positive evaluations. At the same time each connected object or device adds to the complexity, something which may reduce the overall usability of the system for our user group (indicated by the fact that they felt it was too much to test both the balance mat, the lights and the games at the same time).

Consider safety explicitly Another specific design challenge has been that persons with walking difficulties need to focus on the environment and not on the screen. Not being required to look at the screen is important for many users in many situations [33], but for our user group it is crucial. Thus the app needs to be designed so that it can be kept in the pocket, or in the hand without the user looking at it, for all use that involves walking. All our mini-games except one, are designed to be played while standing still. Even so, the rotation involved in the pointing turned out to be challenging for a person with balance problems. This could be beneficial since it implies playing the game will train your balance, but it could also be a problem for persons with significant balance difficulties. The walking faster game, the game which involves walking, is designed so that all information needed to play the game is presented through sound and vibration to allow the user to know what is happening without looking at the screen. A specific problem has been the triggering of the mini-games. For safety reasons, we don't want to risk accidental triggering (which could be the case if shaking/ gestures were used), and require the user to actually touch a location on screen (again, a general screen touch more easily triggers accidentally). This does create problems for persons with very limited fins motor ability. The settings allow for getting the star to the left or the right (to make it easier to touch with either hand during one handed use), but how to safely trigger games while walking is something that requires further work in order to make it accessible for an even wider user group.

A problem with pocket use, has turned out to be accidental touch events (particularly in damp weather). Since the app relies mostly on gestures, and less on touch, the main effect of this has been that the app sometimes can turn off in the pocket without the user realizing this has happened. To prevent such accidental closing of the game, the app currently notifies the user through sound and vibration and also requests extra confirmation before exiting.
Consider how to design for social use We have had very few requests for the sharing of results, in agreement with [10] which stated social support should be optional. At the same time the successful focus group, shows another type of social usage-participants don't share results via social media, but do a joint activity where they all play the same game, and interact while doing so. It is important that games for this group works in these types of group activities-in future work we plan to incorporate and design for such social activities, where the technology is only a part, more explicitly when designing for stroke survivors.

One thing we have struggled with during the development has been finding testers. While it is fairly easy to find participants willing to test more finished products, it has been a struggle to get participants willing to engage in prototype testing. A potential solution here could be to do more of the testing as social activities (e.g. the focus group), and potentially also use these for recruiting individual testers since it might be easier to get testers once they have had a chance to try things out during a focus group. While we used focus groups in the initial stages of the design process, once we had a design possible to test in the home, we switched to individual test activities. In retrospect, this switch was done too soon. Another issue has been to find the right kind of test persons. In our case this doesn't just deal with "playertypes" [34], but also with the consequences of the stroke. The app was intended for persons who were using a smartphone (iPhone) but who found existing games too hard, but the beta testers who did volunteer were quite able, and were in fact already using games like PokemonGo. A lesson here is that for this kind of development, the researcher needs to take a more active role compared to traditional beta testing, and organize more formal social activities (e.g. focus groups/focus tests) in order to get feedback for the development, but also in order to recruit testers. Relying on more social activities, isn't without its own problems however, it is quite possible that the positive feedback at the last focus group was influenced by the fact that the weather was sunny, the participants enjoyed the group activity, and the social atmosphere was nice and friendly (e.g. courtesy bias [35], halo effect [36] etc.).

\section{Conclusion}

We conclude that a wider range of different apps and games suitable for stroke survivors need to be developed-there will be no "silver bullet" game that will appeal to all stroke survivors. Designing for this group is challenging, and developed apps and games need to be flexible and possible to personalize.

Lessons learned: 
- Support varying user abilities, and keep in mind that the abilities of the same user can change from day to day

- Taste varies - if possible, allow aesthetics to be personalizeable

- Design for multimodality

- Avoid sensory overload and limit complexity

- Consider safety explicitly-for a walking game: don't require users to interact with the screen while walking. Avoid time pressure, allow the user to decide when to interact.

- Consider how to design for social use, and take an active role organizing social activities as part of the testing (both for feedback and for recruiting test users)

Future work That different types of activity related apps and technology can increase physical activity in the short term also for stroke survivors is shown both in [16] (increased step count) and our own feasibility study [32] (improved balance). Both studies are too short to say anything about long term adoption. It is known that a third of the users stop using activity trackers within 6 months [37]. Reasons for abandonment have been studied [38], but it is interesting to note that the notion of "player type" [34] doesn't seem to have entered the equation yet. We have informally noted quite big differences in this respect in our users, and feel this is an area of future interest. Related to this is the question of how important activity tracking is in comparison with the tracking of game results. Responses from our alpha tester implies both are important in their own way; you want to follow your activity not just during the game, but over the whole day, but it is also important to be able to follow your game progress (when leveling was thought "unfair", this reduced motivation). Our other testers, however, have not commented on this, and it is a topic which should be further explored.

The game as it is (with the addition of a wizard for the settings) will be released to the app store, and we plan to gather feedback from use "in the wild". It is also our hope our lessons learned, and the game itself, can serve as inspiration for further development of apps and games in this field.

Acknowledgements Open access funding provided by Lund University

Open Access This article is licensed under a Creative Commons Attribution 4.0 International License, which permits use, sharing, adaptation, distribution and reproduction in any medium or format, as long as you give appropriate credit to the original author(s) and the source, provide a link to the Creative Commons licence, and indicate if changes were made. The images or other third party material in this article are included in the article's Creative Commons licence, unless indicated otherwise in a credit line to the material. If material is not included in the article's Creative Commons licence and your intended use is not permitted by statutory regulation or exceeds the permitted use, you will need to obtain permission directly from the copyright holder. To view a copy of this licence, visit http://creativecommons.org/licenses/by/4.0/.

\section{References}

1. Wolf PA, D'Agostino RB, Belanger AJ, Kannel WB (1991) Probability of stroke: a risk profile from the Framingham Study. Stroke 22(3):312-318

2. "research2guidance's mHealth App Developer Economics 2016," no. November, pp 0-4 (2015)

3. Safavi K, Ratli R, Webb K, MacCracken L (2016) Patients want a heavy dose of digital

4. Lazar A, Koehler C, Tanenbaum J, Nguyen DH (2015) Why we use and abandon smart devices. In: Proceedings of the 2015 ACM international joint conference on pervasive and ubiquitous computing, UbiComp 2015

5. Karner J (2018) Most entertaining running and walking apps for Android in 2018. https://www.androidcentral.com/interactive-fitne ss-apps-worth-checking-out. Accessed 25 April 2018

6. Kissela BM et al (2012) Age at stroke: temporal trends in stroke incidence in a large, biracial population. Neurology 79:1781-1787

7. Magnusson C, Rassmus-gröhn K, Rydeman B, Caltenco H (2018) Walk after stroke: initial development of a step counting game for stroke survivors. In: MobileHCI'18 Proceedings of the 20th international conference on human-computer interaction with mobile devices and services adjunct, pp 237-244

8. Magnusson C, Caltenco H, Ólafsdóttir SA (2019) Designing activity games for stroke survivors. In: HAID'19 International workshop on haptic and audio interaction design

9. Lin JJ, Mamykina L, Lindtner S, Delajoux G, Strub HB (2006) Fish 'n'Steps: encouraging physical activity with an interactive computer game. Ubiquitous Comput 2006:261-278

10. Klasnja P, Consolvo S, McDonald DW, Landay JA, Pratt W (2009) Using mobile and personal sensing technologies to support health behavior change in everyday life: lessons learned. AMIA Annu Symp Proc 2009:338-342

11. Consolvo S, McDonald DW, Landay JA (2009) Theory-driven design strategies for technologies that support behavior change in everyday life. In: Conference on human factors in computing systems, pp 405-414

12. Teixeira PJ, Carraça EV, Markland D, Silva MN, Ryan RM (2012) Exercise, physical activity, and self-determination theory: a systematic review. Int J Behav Nutr Phys Act 9(1):1

13. Givon N, Zeilig G, Weingarden H, Rand D (2015) Video-games used in a group setting is feasible and effective to improve indicators of physical activity in individuals with chronic stroke: a randomized controlled trial. Clin Rehabil 30(4):383-392

14. Guo J, Smith T, Messing D, Tang Z, Lawson S, Feng JH (2014) ARMStrokes: a mobile app for everyday stroke rehabilitation. In: ASSETS' 15 Proceedings of the 17 th international ACM SIGACCESS conference on computers and accessibility, vol 1, pp 429-430

15. LeBlanc AG, Chaput JP (2017) Pokémon Go: a game changer for the physical inactivity crisis? Prev Med 101:235-237

16. Paul L et al (2016) Increasing physical activity in stroke survivors using STARFISH, an interactive mobile phone application: a pilot study. Top Stroke Rehabil 23:170-177

17. Donker V, Markopoulos P, Bongers B (2015) REHAP balance tiles: a modular system supporting balance rehabilitation. In: Proceedings of the 9 th international conference on pervasive computing technologies for healthcare 
18. Effenberg AO (2007) Movement sonification: motion perception, behavioral effects and functional data. In: Proceedings of the 2 nd international workshop on interactive sonification

19. Stienstra J, Overbeeke KCJ, Wensveen S (2011) Embodying complexity through movement sonification: case study on empowering the speed-skater. In: Proceedings of the 9th ACM SIGCHI Italian chapter international conference on computerhuman interaction: facing complexity, pp 39-44

20. Feltham F, Loke L (2014) The slow floor: increasing creative agency while walking on an interactive surface. In: Proceedings of the 8th international conference on tangible, embedded and embodied, pp 105-112

21. Srinivasan P, Birchfield D, Qian G, Kidané A (2005) A pressure sensing floor for interactive media applications. In: ACE'05: Proceedings of the 2005 ACM SIGCHI international conference on advances in computer entertainment technology, vol 265, pp 278-281

22. Thornberg K, Josephsson S, Lindquist I (2014) Experiences of participation in rhythm and movement therapy after stroke. Disabil Rehabil 36(22):1869-1874

23. Hadi R, Valenzuela A (2019) Good vibrations: consumer responses to technology-mediated haptic feedback. J Consum Res 47:256-271

24. Caltenco HA, Larsen HS (2014) Designing for engagement: tangible interaction in multisensory environments. In: NordiCHI'14 Proceedings of the 8th Nordic conference on humancomputer interaction: fun, fast, foundational, pp 1055-1058

25. Vandermaesen M, De Weyer T, Luyten K, Coninx K (2014) PhysiCube: providing tangible interaction in a pervasive upper-limb rehabilitation system. In: Tei'14, no. c, pp 1-8

26. Olafsdottir SA et al (2020) Developing ActivABLES for community-dwelling stroke survivors using the Medical Research Council framework for complex interventions. BMC Health Serv Res 20(1):1-14

27. Rassmus-Gröhn K, Magnusson C, Rydeman B, Randall G, Belson $S$ (2017) Conjuring up new technology-using magic objects in co-ideation with stroke survivors, vol 242

28. Magnusson C et al (2017) Tangible interaction for stroke survivors: design recommendations. In: TEI 2017-Proceedings of the 11th international conference on tangible, embedded, and embodied interaction

29. Magnusson C et al (2011) Navigating the world and learning to like it: mobility training through a pervasive game. In: Mobile HCI 2011-13th International conference on human-computer interaction with mobile devices and services

30. Froehlich J et al (2009) UbiGreen: investigating a mobile tool for tracking and supporting green transportation habits. In: Conference on human factors in computing systems-proceedings

31. Dubus G, Bresin R (2013) A systematic review of mapping strategies for the sonification of physical quantities. PLoS ONE 8:e82491

32. Magnusson C et al (2019) Designing motivating interactive balance and walking training for stroke survivors. In: ACM's international conference proceedings series, pp 327-333

33. Magnusson C, Larsson A, Warell A, Eftring H, Hedvall P-O (2012) Bringing the mobile context into industrial design and development. In: NordiCHI 2012: making sense through designProceedings of the 7th Nordic conference on human-computer interaction

34. Hamari J, Tuunanen J (2012) Player types: a meta-synthesis Juho. In: Proceedings of Nordic DiGRA 2012 conference games in culture and society, pp 29-53

35. Quinton S (2015) "Ciccarelli SK, Noland White J, Psychology, 4th edn/Global edition". In: Psychology learning and teaching

36. Baron J (2006) Thinking and deciding

37. Hammond $\mathrm{T}$ (2014) Wearables have a dirty little secret: $50 \%$ of users lose interest. Tech Republic Inc, Louisville

38. Attig C, Franke T (2020) Abandonment of personal quantification: a review and empirical study investigating reasons for wearable activity tracking attrition. Comput Human Behav 102:223-237

Publisher's Note Springer Nature remains neutral with regard to jurisdictional claims in published maps and institutional affiliations. 